\title{
Reinforcing password authentication with typing biometrics
}

\author{
W.G. de Ru, J.H.P. Eloff \\ Dept. Computer Science, Rand Afrikaans University, P.O. Box 524, Auckland Park, 2006, \\ South-Africa
}

\begin{abstract}
This paper presents a methodology for the reinforcement of password authentication with the use of typing biometrical mechanisms. Typing biometrics refers to the analysis of keystroke patterns, which is unique for each user. The analysis of these keystroke patterns is done transparently, without being noticeable to the user, while he/she tries to gain system access via a password authentication mechanism in the normal way, i.e. by entering a user identification and password string. The analysis of keystroke patterns is done with fuzzy logic techniques, which are shown to have great potential in uniquely categorising each user's unique typing patterns. Fuzzy logic techniques have proven to be very successful in a wide range of applications, with much commercial success. It is shown that the integration of fuzzy logic based typing biometrics into currently employed password mechanisms, which is the most commonly used authentication device, can only serve to enhance security.
\end{abstract}

Keywords: Computer Security, Authentication, Biometrics, Keystroke dynamics, Fuzzy logic, Authentication, Passwords

\section{INTRODUCTION}

The successful verification of a user or entity wishing to utilise a computer system lays at the core of the security of these systems. Effective system administration, auditing and efficient resource management all depend on correct user identification[JOBU89, PFLE93, SPEN87]. Although a variety of different authentication devices are in use today to establish the identity of a user, password mechanisms remain the predominant method of choice. Password authentication is an inexpensive, familiar paradigm, which is supported by most operating systems[CONN90, SPEN87]. Confidence in its ability to provide adequate authentication, however, is diminishing [CONN90, SPEN87]. This can mainly be attributed to the poor usage of passwords by many users. Methods are needed to extend and enhance the useful life of password techniques. In this paper discussion focuses on the use of typing biometrics as a reinforcing mechanism for authentication usually achieved with password techniques. Typing biometrics refers to the analysis of a user's keystroke patterns[CONN90]. This reinforcing mechanism is transparently employed without being noticeable to the user while he/she enters the normal authentication information, i.e. the user identification and password.

Each user has an unique way of using the keyboard to enter his/her password, e.g. each user types the different characters that compose the password, at different typing speeds. Fuzzy logic 
techniques are used to measure the typing biometrics of a user. Fuzzy logic is the process of modelling problems that deal with ambiguous data using multivalued logic[BARR93, JOHN90, KOSK92, KOSK93, ZADE65]. It's a generalisation of the traditional bivalent logic, which states that any assertion can either be true or false, but not both simultaneously. The statement: "the time interval between typing two successive password characters is very fast", is ambiguous, where can the line for "very fast" be drawn? Fuzzy logic holds that everything is a matter of degree, e.g. the typing speed between typing two successive password characters might belong $90 \%$ to the set of fast typing.

Currently, the quantity of commercially available equipment utilising fuzzy logic is proliferating at a tremendous pace[BARR93, JOHN90, KOSK93, MCNE94]. It is finding usage in fields as diverse as mission critical train control where many lives are at stake, to the control of a normal washing machine.

To describe the mechanisms for integrating typing biometrics into password authentication, the remainder of this paper is structured as follows:

Section 2 briefly discusses authentication and the mechanisms in use.

Section 3 discusses the advantages and disadvantages of the different authentication mechanisms and argues the use of biometrics combined with passwords.

Section 4 presents the framework for the implementation of typing biometrics.

Finally the advantages, as well as further research, are discussed in section 5.

\section{AUTHENTICATION}

The correct identification of a user is at the heart of most security systems. The identity of a user is necessary to maintain access control, as well as accountability[JOBU89]. A variety of different authentication devices are in use today to establish the identity of a user.

Authentication techniques are grouped into three main categories[DERU93, MENK88, PFLE93]. The first authentication category requires the possession of an object. Smart cards and magnetic strip cards are examples thereof[SPEN87]. The second category entails that a user supplies specific information or responses to questions. Passwords are examples of authentication devices in this category[JOBU89]. The third category requires that the authentication device measures some physical characteristic of the person being verified[CONN90, JOBU89, SHER92]. This includes biometrical mechanisms such as face recognition, fingerprints, voiceprints, retina scans, keystroke patterns and signatures. In the strictest sense, biometrics refers to the use and application of statistical analysis to biological data and phenomena[OXFO90]. It has, however, become widely accepted within the security community to describe technologies used in personal identity verification[SHER92]. The biometrical devices can be further devided into two main categories: Those devices that use physical characteristics such as fingerprints and hand geometry, and those devices that use behavioural characteristics such as signature dynamics and keystroke dynamics[SHER92].

All authentication devices, as discussed above, endeavour to eliminate the positive validation of an intruder(Type II error), as well as the failing to positively validate a legitimate user(Type I error). They further strive to impose a minimal extra burden on users, whilst still avoiding type I and II errors. Finally, they attempt to provide authentication at a reasonable cost[DERU93, JOBU89, PFLE93]. 


\section{AUTHENTICATION: TOWARDS A BETTER SOLUTION}

Associated with each of the above mentioned authentication categories, are also inherent strengths and weaknesses. The authentication devices that require the possession of an object, are susceptible to loss or theft, e.g. magnetical strip cards are vulnerable to being copied by using relatively cheap equipment. The advantages of these devices are the high level of security they provide[SPEN87].

The authentication devices that entail the supplying of specific information such as a password, are very vulnerable to trial and error attacks because users normally have difficulty in choosing passwords that are simultaneously memorable and difficult to guess. The infamous Internet worm of 1989 , for example, used a commonly used password dictionary to compromise the security of many of the network sites[SEEL89]. These devices, however, are still the cheapest and also the most widely used.

Because the authentication information utilised by the devices requiring the measuring of some physical characteristic, i.e. biometrical techniques, can't be forgotten or stolen, it seems a very attractive solution. The inability of these devices however, to completely eliminate type I and II errors, combined with their expensive price tag, hampers their coming of age.

All authentication devices, to a bigger or lessor extent, are susceptible to type I and type II errors. No device can be completely relied on to eliminate the validation of an intruder and the denying of access to a legitimate user. No single mechanism or control on its own, can be trusted to provide completely adequate security. It has justly been said that computer security should be composed of a combination of locks and control mechanisms[RUSS91]. The most effective defence will be composed of a sequence of interrelated security mechanisms. In this manner each vulnerability in a security function is balanced by another security mechanism[RUSS91]. The same rationale also applies to authentication mechanisms. If different authentication devices could be used in combination, while acknowledging the fact that costs must be kept manageable and a minimal extra burden must be imposed on users, the occurrence of type I and II errors could be kept to a minimum. Authentication will be optimised if mechanisms are synergistically combined, so that the sum of the whole can be greater than the sum of it's parts.

The remainder of this paper explores a methodology that integrates typing biometrics into current password authentication mechanisms as a means of improving security and reducing type I and II errors. The typing biometrics methodology employs a software solution in measuring a user's biometrical characteristics, which is a cheap alternative that keeps costs manageable, but still maintains the benefits inherent to biometrics, i.e. that it can't be forgotten, forged or stolen.

\section{REINFORCED PASSWORDS}

A brief overview of the methodology is given to serve as frame of reference for the subsequent discussions.

If a new user requests to be allowed access the computer system, or if an existing user's password expires, he/she will be asked to type a user identification and a new password. The user will then be asked to re-enter the user identification and password to verify the previous inputs. Based on the typing patterns displayed on entering and re-entering the user identification and password, the typing biometrics methodology will compute a typing index for the user. The 
authors are aware that the number of digits in the user identification and password will have an important impact on the establishing of an accurate pattern. A user identification with an associated typing index will be saved by the access control system, along with the normal user identification-password pair.

On subsequent attempts to gain access to the system, the user will have to go through the normal password authentication procedure, i.e. entering the user identification followed by the password. Simultaneously, the system will also monitor the user's typing patterns and compute a typing index based on the user identification and password pair just entered. This typing index will be compared with the typing index previously determined for this user. This will transparently be done without being noticeable to the user. If both the password and typing index match those saved by the authentication mechanism, a user is allowed access to the system. A typical session when a user attempts to access a system, is illustrated in figure 1.

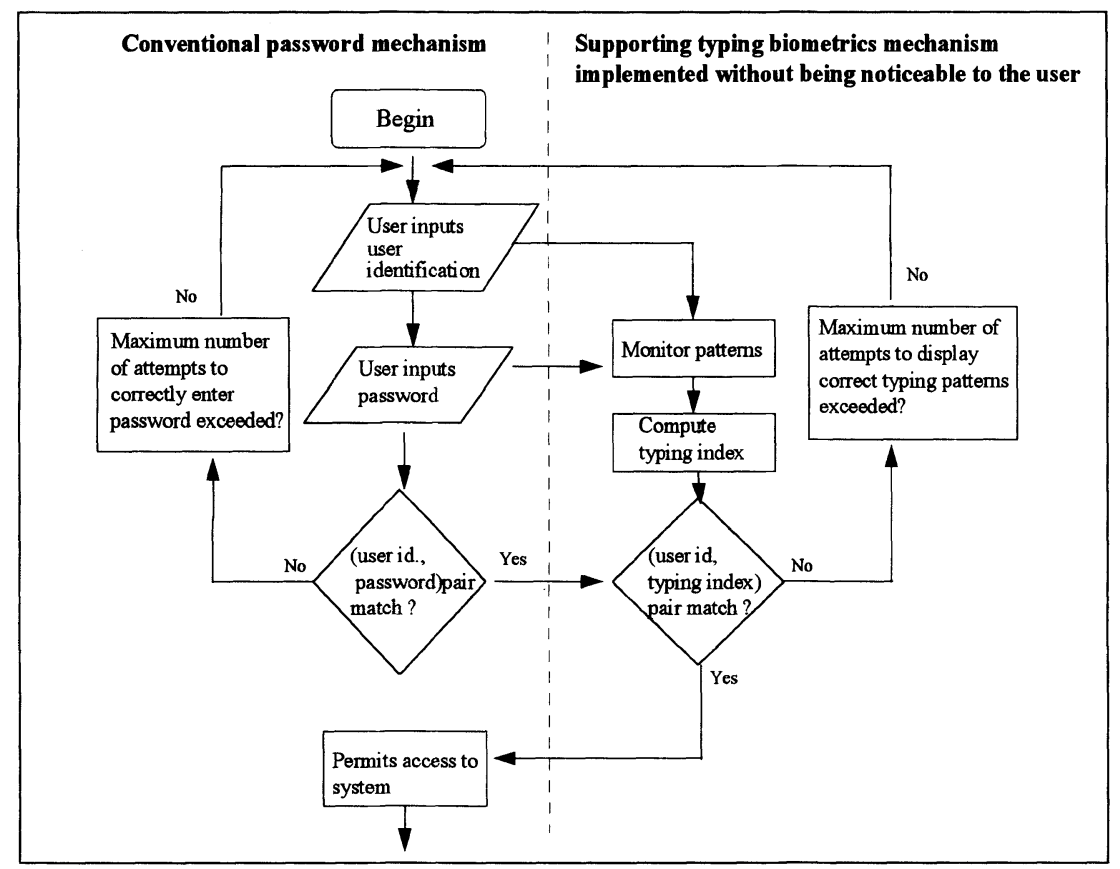

Figure 1 Reinforced password authentication with typing biometrics

The reinforcing typing biometrics component of the methodology, which categorises users' keystroke patterns, is now discussed, with special attention given to the following:

Firstly, the output which is produced to uniquely categorise each user's typing patterns, is presented.

Secondly, the inputs used to produce an unique categorisation for each user, are explained. Thirdly, it is shown how the output, i.e. the unique categorisation, is influenced by different inputs. 
Relating precise values to fuzzy sets is explained next, whereafter the categorisation with the use of rules is shown.

Finally, the way in which authentication is achieved with this methodology, is explained.

\subsection{System output}

Because the aim of the methodology is to uniquely categorise each user's typing patterns, the output will be the categorisation of these patterns. It is important that each user's typing patterns are uniquely categorised, the reason being that this categorisation, along with a password, will be the differentiating criteria by which a user is identified.

\subsection{System inputs}

The typing biometrics methodology as proposed in this paper utilises two inputs to categorise a user's keystroke patterns. Firstly, it utilises the time intervals between successive characters[OBAI93], created by users while typing a known sequence of characters, i.e. the user identification and password. Secondly, it utilises the "typing difficulty" in typing these successive characters. Literature and study on typing patterns suggest that certain key combinations on a conventional typewriter or computer keyboard are more difficult to perform that others[WEST69]. For the purposes of this paper the typing difficulty is defined as the number of physical keys on the keyboard separating the successive characters entered by the user, as well as a component that determines whether the character was created with alternative key combinations, e.g. typing a capitalised character normally entails pressing two keys simultaneously.

These inputs will be illustrated with the following example: Suppose that the user enters Jack as user identification and $B e a N$ as password. The first input, i.e. the time interval, will entail the time duration between the letter pairs (J,a), (a,c), (c,k), (k, "Enter"), ("Enter", B), (B,e), (e,a), $(a, N)$ measured in milliseconds. The second input, i.e. the typing difficulty, will entail the determination of the number of physical keys on the keyboard separating these letter pairs, combined with an added component that determines whether alternative key combinations were used, e.g. the letter pair $(\mathrm{a}, \mathrm{N})$ will yield a typing difficulty of 10 because 5 physical keys separate the "a" and " $n$ " on the keyboard and the " $n$ " was typed in combination with the "shift" key to produce a capital " $N$ ", therefore adding an additional weight to the eventual typing difficulty factor. For simplicity of discussions, the value chosen for the weight is 5 , but can easily be adapted to fine-tune the different results or categorisations obtained by the methodology.

The inputs and output utilised by the methodology are summarised in Table 1.

Table 1

Inputs and Output

\begin{tabular}{ll}
\hline Inputs & Output \\
\hline time interval & categorisation \\
typing difficulty & \\
\hline
\end{tabular}

\subsection{Associating the inputs with the output}

The inputs influence the output, i.e. the time interval and the typing difficulty influence the way in which a user will be categorised. There is a relationship between the inputs and the output, but it is vague or fuzzy. It is difficult to crisply quantify the effect that the inputs will have on the output. It is, however, possible to vaguely express the relationships in linguistic terms, e.g. it 
is possible to say that if a time interval was very slow and if it was not difficult to type, the user must be categorised as typing poorly. The linguistic expressions represent subsets of the inputs and output utilised, e.g. not difficult is a subset of the complete set of different typing difficulties. To be able to relate the inputs to the output, therefore, subsets must be defined for each of the inputs, as well as for the output.

Different subsets are possible for each of the above inputs. The time interval between successive characters could possibly be very slow for a user not acquainted with a computer keyboard, or very fast for an experienced user. The following sets are used by the typing biometrics methodology: Very slow, Slow, Medium, Fast and Very fast. Likewise, the typing difficulty of typing successive characters could be very easy for typing a $(a, c)$ pair, or more difficult for typing a $(\mathrm{a}, \mathrm{N})$ pair. The following sets are used by the typing biometrics methodology: Very easy, Easy, Difficult and Complex.

Similarly, it is also necessary to define subsets for the output. The categorisation of a keystroke pattern could possibly be very bad for typing patterns displayed by an inexperienced user or excellent for typing patterns displayed by an experienced user. The following sets are used by the typing biometrics methodology: Very bad, Bad, Below average, Average, Good, Very good and Excellent.

With the use of the above defined subsets, the relationship between the inputs and output can now be expressed as follows:

\section{Rule 1: IF TIME INTERVAL IS VERY FAST AND TYPING DIFFICULTY IS EASY THEN CATEGORISE AS VERY GOOD \\ Rule 2: IF TIME INTERVAL IS VERY SLOW AND TYPING DIFFICULTY IS VERY EASY THEN CATEGORISE AS VERY BAD \\ Rule 3: IF TIME INTERVAL IS MEDIUM AND TYPING DIFFICULTY IS DIFFICULT THEN CATEGORISE AS AVERAGE \\ Rule 4: IF TIME INTERVAL IS SLOW AND TYPING DIFFICULTY IS EASY THEN CATEGORISE AS BELOW AVERAGE \\ Rule 5: IF TIME INTERVAL IS MEDIUM AND TYPING DIFFICULTY IS COMPLEX THEN CATEGORISE AS GOOD \\ Rule 6: IF TIME INTERVAL IS FAST AND TYPING DIFFICULTY IS COMPLEX THEN CATEGORISE AS GOOD \\ Rule 7: IF TIME INTERVAL IS VERY FAST AND TYPING DIFFICULTY IS COMPLEX THEN CATEGORISE AS EXCELLENT}

Rule 8: IF TIME INTERVAL IS FAST AND TYPING DIFFICULTY IS DIFFICULT THEN CATEGORISE AS GOOD

More rules are utilised in the complete prototype, but for the sake of the simplicity of explanations, these rules are used.

\subsection{Relating precise values to fuzzy sets}

Although it is possible to express the relationships between the inputs and the output in linguistic terms, the inputs utilised by the typing biometrics methodology are precise, although not always the same, e.g. the time interval for the same user who types a $(J, a)$ pair could possibly measures 0.58 milliseconds, or sometimes possibly 0.6 milliseconds, which are precise values, despite the fact that it will not always be exactly the same.

These precise values, such as 0.58 milliseconds or 0.6 milliseconds, must be related to linguistic sets to be able to utilise the linguistic rules as defined above. This is done with the use of membership functions. Figures 2 and 3 illustrate the membership functions for the time interval 
and typing difficulty inputs. Figure 4 illustrates the membership functions for the categorisation output. A membership function refers to the degree to which a specific precise value belongs to a set[KOSK92, KOSK93, MCNE94]. A time interval of 0.58 milliseconds in figure 2 for example, belongs $20 \%$ to the set Fast and $70 \%$ to the set Medium. Similarly, a typing difficulty factor of 10 in figure 3, belongs $15 \%$ to the set Complex and $70 \%$ to the set Difficult. From the examples it can be seen that it is possible for a value to simultaneously belong to more than one set. Subsequent discussions will illustrate how this feature of fuzzy logic harmonises all obtained inputs, even if these inputs are not exactly the same for each authentication session, to produce an unique categorisation.

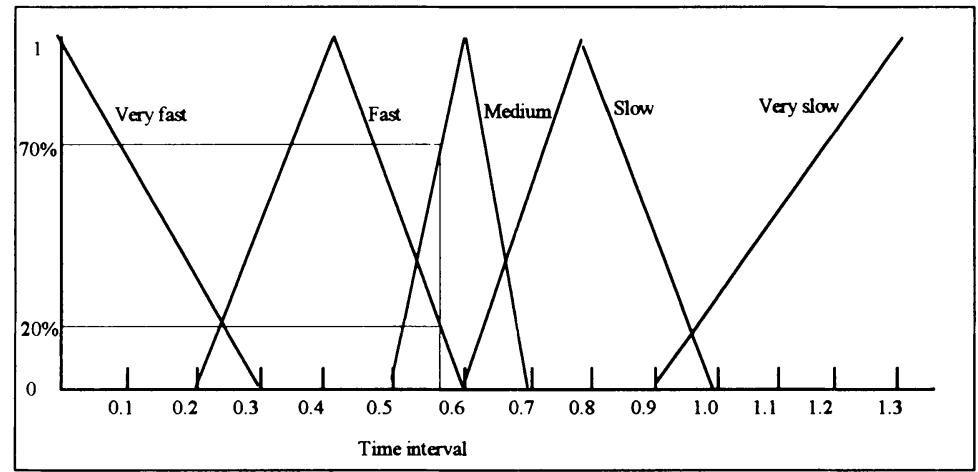

Figure 2 The time interval input



Figure 3 The typing difficulty input

\subsection{Categorisation}

When evaluating a user's keystroke patterns, the time interval and typing difficulty input values cause certain rules to be activated by the typing biometrics methodology, e.g. input values that fall in the medium and difficult sets will activate rule 3 because rule 3 states that if time interval is medium and typing difficulty is difficult then categorise as average. Rules are activated simultaneously in a fuzzy system[KOSK92, KOSK93]. This is possible because an input value can simultaneously belong to more than one set. When a rule is activated it is referred to as being fired. The way in which inferencing by the methodology is achieved will best be illustrated by an example. 


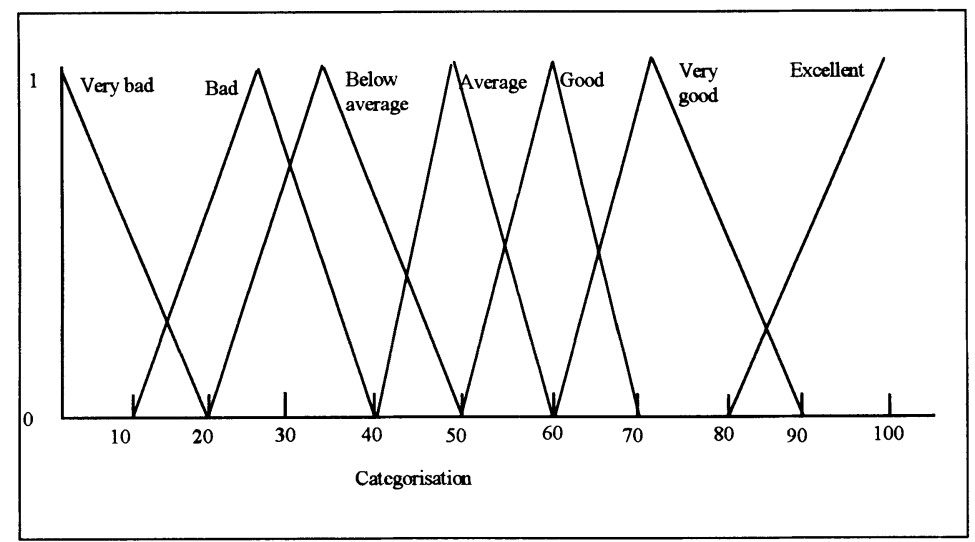

Figure 4 The categorisation output

Assume that one of the character pairs that the user entered was $(\mathrm{a}, \mathrm{N})$, which yielded a time interval of 0.58 milliseconds and a typing difficulty of factor 10. It is shown in figures 2 and 3 to which fuzzy sets these specific input values belong. The time interval input of 0.58 milliseconds belongs to both the sets Medium and Fast. Similarly, the typing difficulty of factor 10 belongs to both the sets Difficult and Complex. The following statements therefore hold: The time interval is medium and fast, and the typing difficulty is difficult and complex. This causes the following rules to fire:

Rule 3: IF TIME INTERVAL IS MEDIUM AND

TYPING DIFFICULTY IS DIFFICULT THEN CATEGORISE AS AVERAGE

Rule 5: IF TIME INTERVAL IS MEDIUM AND

TYPING DIFFICULTY IS COMPLEX THEN CATEGORISE AS GOOD

Rule 6: IF TIME INTERVAL IS FAST AND

TYPING DIFFICULTY IS COMPLEX THEN CATEGORISE AS GOOD

Rule 8: IF TIME INTERVAL IS FAST AND

TYPING DIFFICULTY IS DIFFICULT THEN CATEGORISE AS GOOD

Because the input values only partially belong to the sets, e.g. 0.58 milliseconds only belongs $70 \%$ to the set Medium, the rules must likewise only fire partially. Figure 5 illustrates how categorisation is achieved with the use of these rules and will be discussed in the remainder of this paragraph. Only the specific sets involved in each rule are shown in the figure.

The vertical lines represent the crisp input values, i.e. the 0.58 milliseconds time interval and a factor 10 typing difficulty. All four rules fire at once. In rule 3, i.e. If time interval is medium and typing difficulty is difficult then categorise as average, the vertical lines indicate a membership of $70 \%$ in both the medium time interval set, as well as the difficult typing difficulty set. This rule, therefore recommends an Average categorisation to a degree of $70 \%$. In rule 5, i.e. If time interval is medium and typing difficulty is complex then categorise as good, the vertical lines indicate a membership of $70 \%$ in the medium time interval and $20 \%$ in the complex typing difficulty. Different methods can be employed in fuzzy logic to perform the AND logical operation[KOSK92, KOSK93, MCNE94]. In the most common approach the rule takes the lesser of $20 \%$ and $70 \%$, which is $20 \%$, and applies it to the output set[KOSK92, KOSK93, MCNE94]. This rule therefore recommends a Good categorisation to a degree of $20 \%$. Likewise, the sixth and eight rules select the smaller of the two memberships and recommend 
categorisation to that extend. The process of firing these fuzzy rules is done for each of the character pairs in the user identification and password string that were entered by the user.

Next, after all the character pairs have been entered by the user and evaluated by the methodology, the recommendations of all the rules that have been fired must be totalled up[MCNE94]. To do this, the methodology truncates the categorisation triangles at the recommendation level, as is normally done in fuzzy logic applications[MCNE94]. It then superimposes the resultant trapezoids on top of each other, to create a new geometrical shape, like the shaded area at the bottom of figure 6 . Finally, the methodology must now resolve all the recommendations into a crisp quantifiable category. Different methods also exist in fuzzy logic theory to achieve

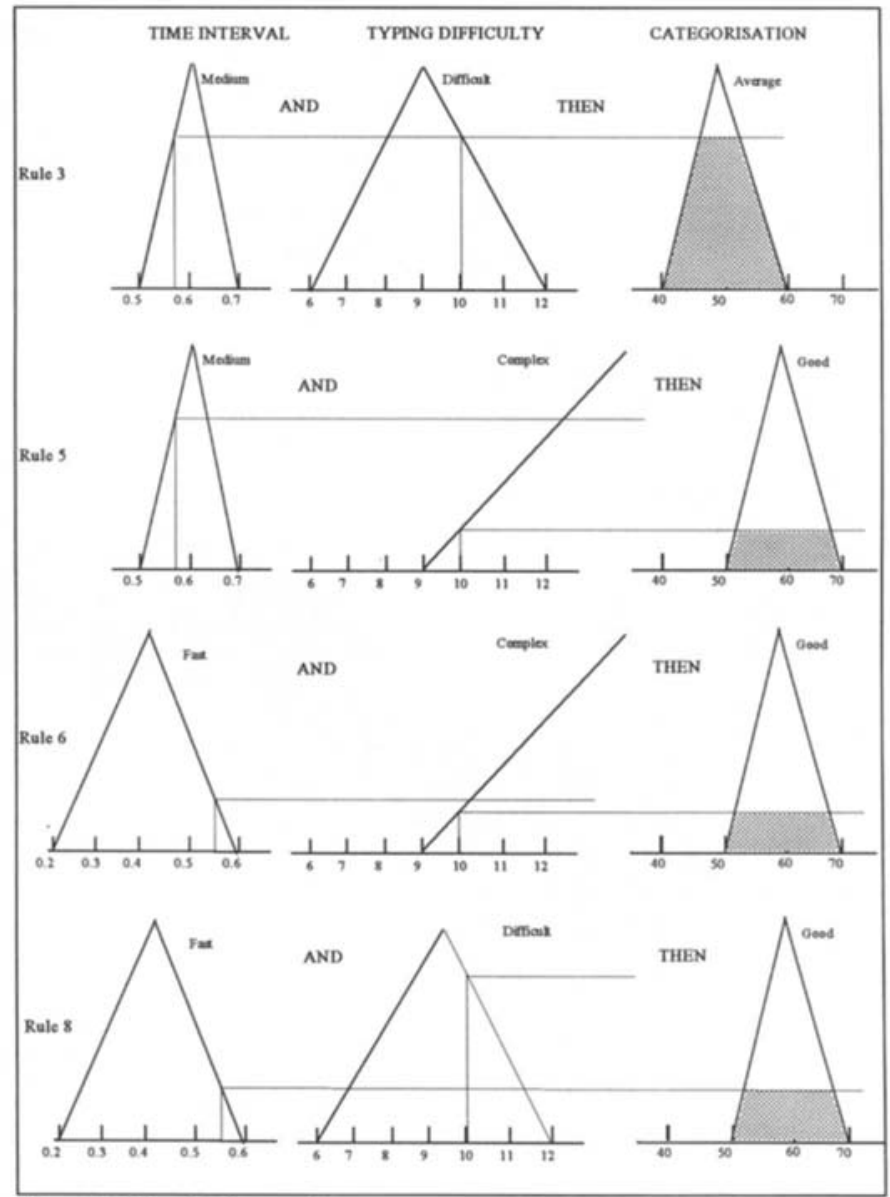

Figure 5 Firing the fuzzy rules 
this[KOSK92, KOSK93, MCNE94]. The most common of these, utilises the centre of gravity of the geometrical shape. This value is crisp and yields a numerical categorisation, i.e. the typing index. In figure 6 for instance, the centre of gravity is somewhere between 50 and 60 and the user will be categorised accordingly.

\subsection{Allowing system access}

The above described mechanisms render a typing index for the specific user trying to gain access to a computer system while analysing the user's typing patterns displayed when entering an user identification and password. The entered password and computed typing index are then compared to those previously saved by the access control mechanism for the specific user identification. If both the password and typing index match those saved by the access control mechanism for the specific user identification, the user is allowed access to the system. If the

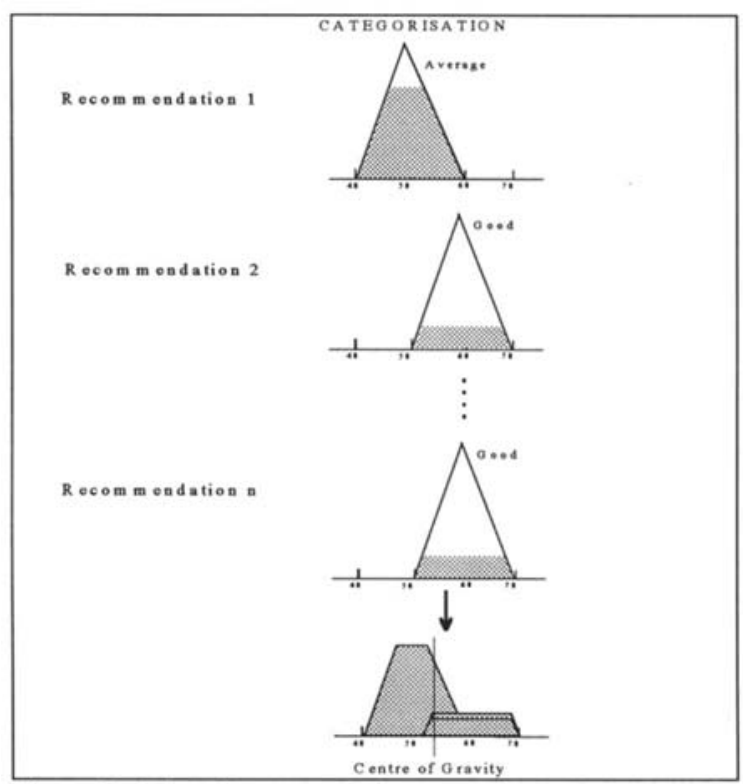

Figure 6 Relating all recommendations to a crisp answer

password does not match, i.e. if it was incorrectly entered, a user will be rejected or asked to reenter the authentication information, by the normal password authentication mechanism, without utilising the biometrics component. If the password does match, i.e. if it was correctly entered, the biometrics component is used as a supporting recommendation that verifies that the person trying to gain access is really who he/she claims to be. 


\section{ADVANTAGES AND FURTHER RESEARCH}

The synergistical reinforcement of password mechanisms with the use of typing biometrics can only serve to improve authentication. The following advantages are inherently part of the methodology as described in this paper:

- Because it does not require expensive hardware, costs are kept manageable.

- A compromised password obtained by an intruder will not necessarily mean that access to the system will erroneously be allowed.

- A user's typing biometrics can't be lost, stolen or lend.

- A user's typing biometrics can't be obtained while peaking at the user's typing.

- The integration into current security systems will be smooth because it can be used in conjunction with the current password mechanisms. The fact that it is software and not hardware driven, further improves the integration possibilities.

- The technique can be used to validate a user trying to gain access from any site or system(even if geographically separated), not only those sites trusted by the system or those equipped with special hardware.

- Trial and error password attacks have effectively been rendered unusable. The rejection of a password does not necessarily provide information pertaining the correctness of the password string.

- Throughout the duration of the session the typing biometrics mechanism can keep on monitoring the keystrokes of the user to ensure that the user initiating the session is still the same user currently using the system.

Research presented in this paper is a first attempt to reinforce passwords with the use of fuzzy logic based typing biometrics, but further research is necessary. The following high-level discussion highlights areas for further investigation:

- The exact scales utilised by the methodology for the typing difficulty input and categorisation output, as well as the optimum calibration of membership functions, to produce as unique a categorisation as possible for each user, need further examination. Also, the deviation allowed when comparing the newly computed typing index with the saved typing index, must be examined. Deviations in matching a specific user's typing index might occur because of the fact that the exact same typing patterns are not always displayed each time the authentication information is entered by a user, e.g. the time interval for a specific character pair could measure 0.58 milliseconds in some instances and in other instances it could possibly measure 0.6 milliseconds. This vagueness or fuzziness in input, is however mostly addressed with the use of fuzzy logic techniques which do not restrict crisp input values, such as 0.58 milliseconds or 0.6 milliseconds, to belong to only one set. Fuzzy logic techniques also utilise the recommendations of all the rules in a harmonised manner to obtain a typing index that does not deviate substantially from the previously computed typing index.

- The reliance which the access control system places on the recommendation of the typing biometrics component, also needs to be further researched. The following are some of the questions that must be addressed: Will the user be denied access if the methodology notices a typing index mismatch? Will the user be allowed access but the security administrator 
notified if the methodology notices a typing index mismatch? Will the user have to re-enter his authentication information if the methodology notices a typing index mismatch?

- Rather then defining the typing difficulty as the physical separation between keys, the theory behind the design of the keyboard for typing efficiency and ergonomics could be used.

- The above proposed research pertaining the scales, calibration and reliance of the typing biometrics component will only be possible by implementing a working model of the methodology, albeit experimental, in an actual password authentication environment, for an extended time period. Questions such as the number of digits in a password that are necessary to give a correct pattern match, and the number of times that the authentication information needs to be entered to establish a pattern also needs further research by means of a working model. Answers obtained to these questions will also answer questions pertaining to the cost and time delay if passwords are changed regularly.

- The use of neural nets combined with fuzzy logic to increase the ability of the system to learn a user's keystroke patterns, are also a potential field for research. This will, for example, be helpful in situations were users who are initially new to a computer environment and display poor typing abilities, will improve their typing performances with regular use of the computer. A user's typing pattern can also change when regularly typing the same authentication information. With the use of neural nets, the authentication mechanism will possibly be able to adapt to a user's changing typing patterns, i.e. adjusting the typing index based on a user's changing typing patterns.

\section{CONCLUSION}

The ongoing research and attention given to authentication mechanisms, is a clear indication of the fact that accurate, user-friendly authentication at manageable costs, remains a somewhat illusive goal. This paper presented an inexpensive methodology that reinforces current password authentication, which is the most widely used authentication mechanism and would remain so for a long time to come. This reinforcing mechanism is implemented in such a manner that the user is unaware of it's presence. In any event, the security yielded by the typing biometrical reinforcing mechanism, can only serve to improve and enhance security achieved by the conventional password mechanism, with which it is integrated. The fact that the typing biometrics methodology integrates into current password mechanisms at manageable costs to decrease the occurrence of type I and II errors, while maintaining the advantages of biometrical devices, makes it a worthwhile approach in the quest for the "ultimate authentication mechanism".

In conclusion it must be said that the techniques for enhancing password security outlined in this paper must never replace the education of users in using the authentication mechanisms. Security can be said to be not only the implementers concern but also the users'.

\section{REFERENCES}

[JOBU89] Jobusch D.L., Oldehoeft A.E., "A survey of Password Mechanisms: Weaknesses and Potential Improvements. Part 1", Computers \& Security, $8(1989)$ pp. 587-604 
[JOBU89] Jobusch D.L., Oldehoeft A.E., "A survey of Password Mechanisms: Weaknesses and Potential Improvements. Part 2", Computers \& Security, 8(1989) pp. pp. 675-689

[OXF090] Oxford Concise Dictionary, Oxford, Clarendon Press Oxford, 1990

[PFLE93] Pfleeger C.P. Security in Computing, Prentice Hall, Englewood Cliffs, 1993

[SPEN87] Spender J.C., "Identifying Computer Users with Authentication Devices (Tokens)", Computers \& Security, 6 (1987), pp. 385-395

[CONN90] Conn A.P., Parodi J.H., Taylor M., "The place of Biometrics in A User Authentication Taxonomy", Proceedings 13th National Computer Security Conference, Washington, Oct. 1990

[BARR93] Barron J.J., Putting Fuzzy Logic into Focus", Byte, April 1993

[JOHN90] Johnson J.T., "Fuzzy Logic", Popular Science, July 1990, pp. 87-89

[KOSK92] Kosko B., "Neural Networks and Fuzzy Systems", Prentice Hall International editions, 1992

[KOSK93] Kosko B., "Fuzzy Thinking The New Science of Fuzzy Logic", Hyperion, 1993

[ZADE65] Zadeh L.A. "Fuzzy sets", Information and Control, vol. 8, pp. 338-353, 1965

[MCNE94] Mc Neil D., Freiberger P., Fuzzy Logic, Touchstone Simon \& Schuster, 1994

[DERU93] de Ru W.G., Eloff J.H.P., "Passphrase generation through expert system technology", Proceedings 9th Annual Computer Security Applications Conference, Orlando, Florida, Dec. 1993

[MENK88] Menkus B. Understanding the use of Passwords, Computers \& Security, 7(1988) pp. 132-136

[SHER92] Sherman R.L., "Biometrics Futures", Computers \& Security, 11 (1992)

[SEEL89] Seeley D., "Password cracking: A Game of Wits", Communications of the $A C M$, June 1989, Volume 32, Number 6

[RUSS91] Russel D., Gangemi G.T., Computer Security Basics, O'Reilly and Associates, Inc., July 1991

[OBAI93] Obaidat M.S., "A methodology for improving computer access security", Computers \& Security 12 (1993) pp 657-662

[WEST69] West J.W., The acquisition of typewriting skill, Pitman Publishers, 1969 